\title{
Synaptic Tagging and Capture: Differential Role of Distinct Calcium/Calmodulin Kinases in Protein Synthesis-Dependent Long-Term Potentiation
}

\author{
Roger L. Redondo, ${ }^{1}$ Hiroyuki Okuno, ${ }^{2}$ Patrick A. Spooner, ${ }^{1}$ Bruno G. Frenguelli, ${ }^{3}$ Haruhiko Bito, ${ }^{2}$ \\ and Richard G. M. Morris ${ }^{1}$ \\ ${ }^{1}$ Centre for Cognitive and Neural Systems, University of Edinburgh, EH8 9JZ, Edinburgh, United Kingdom, ${ }^{2}$ Department of Neurochemistry, University of \\ Tokyo Graduate School of Medicine, Bunkyo-ku, Tokyo 113-0033, Japan, and ${ }^{3}$ Department of Biological Sciences, University of Warwick, CV4 7AL, \\ Coventry, United Kingdom
}

Weakly tetanized synapses in area CA1 of the hippocampus that ordinarily displaylong-term potentiation lasting $\sim 3 \mathrm{~h}$ (called early-LTP) will maintain a longer-lasting change in efficacy (late-LTP) if the weak tetanization occurs shortly before or after strong tetanization of an independent, but convergent, set of synapses in CA1. The synaptic tagging and capture hypothesis explains this heterosynaptic influence on persistence in terms of a distinction between local mechanisms of synaptic tagging and cell-wide mechanisms responsible for the synthesis, distribution, and capture of plasticity-related proteins (PRPs). We now present evidence that distinct CaM kinase (CaMK) pathways serve a dissociable role in these mechanisms. Using a hippocampal brain-slice preparation that permits stable long-term recordings in vitro for $>10 \mathrm{~h}$ and using hippocampal cultures to validate the differential drug effects on distinct CaMK pathways, we show that tag setting is blocked by the CaMK inhibitor KN-93 (2-[N-(2-hydroxyethyl)]- $N$-(4-methoxybenzenesulfonyl)amino- $N$-(4-chlorocinnamyl)$\mathrm{N}$-methylbenzylamine) that, at low concentration, is more selective for CaMKII. In contrast, the CaMK kinase inhibitor STO-609 [7Hbenzimidazo(2,1-a)benz(de)isoquinoline-7-one-3-carboxylic acid] specifically limits the synthesis and/or availability of PRPs. Analytically powerful three-pathway protocols using sequential strong and weak tetanization in varying orders and test stimulation over long periods of time after LTP induction enable a pharmacological dissociation of these distinct roles of the CaMK pathways in late-LTP and so provide a novel framework for the molecular mechanisms by which synaptic potentiation, and possibly memories, become stabilized.

\section{Introduction}

Activity-dependent synaptic plasticity, such as long-term potentiation (LTP) (Bliss and Lomo, 1973), is widely thought to be involved in the encoding of new information during learning. Some forms of LTP decay to baseline over a short timescale (early-LTP), whereas others, notably late-LTP, show stable synaptic potentiation for much longer (Krug et al., 1984; Frey et al., 1988). The synaptic tagging and capture (STC) hypothesis of late-LTP (Frey and Morris, 1997) asserts that LTP involves the local "tagging" of synapses at the moment of induction, that tags capture diffusely transported "plasticity-related proteins" (PRPs) synthesized in the soma or local dendritic domains, and that tag-PRP interactions are essential for stabilization of potentiation. Various lines of evidence support this framework (Frey and

Received June 10, 2009; revised Dec. 15, 2009; accepted Jan. 6, 2010.

This work was supported by grants from the Human Frontier Science Program (R.G.M.M., H.B.), Volkswagen Stiftung (R.G.M.M.), the U.K. Medical Research Council (R.G.M.M.), the Ministry of Education, Culture, Sports, Science and Technology and Ministry of Health, Labor, and Welfare of Japan (H.B., H.O.), the Takeda Foundation (H.B.), and the Yamada Science Foundation (H.B.). We thank Tobias Bonhoeffer, Rosalina Fonseca, Mark van Rossum, and members of the Laboratory for Cognitive Neuroscience in Edinburgh for discussion and Feruza Nuritova and Colin McKenzie for earlier data.

Correspondence should be addressed to R. G. M. Morris, Centre for Cognitive and Neural Systems, University of Edinburgh, 1 George Square, EH8 9JZ, Edinburgh, UK. E-mail: r.g.m.morris@ed.ac.uk.

DOI:10.1523/JNEUROSCI.3140-09.2010

Copyright $\odot 2010$ the authors $\quad 0270-6474 / 10 / 304981-09 \$ 15.00 / 0$
Morris, 1997; Martin et al., 1997; Reymann and Frey, 2007), but important open questions concern the signal-transduction pathways involved in tag setting and PRP availability.

Because relevant molecular interactions are likely to take place over several hours after LTP induction, the short time course of most LTP experiments ( $1-3 \mathrm{~h}$ ) may be insufficient to monitor all the processes involved in synaptic stabilization. We therefore developed long time course in vitro protocols that involve (1) an interaction of strong tetanization (that sets tags and upregulates PRP synthesis) and temporally adjacent weak tetanization (which only sets tags), and (2) the rapid onset and cessation of selective kinase inhibition at appropriate times. The successful cessation of inhibition requires both rapid drug washout (i.e., the drug does not permanently bind to its target) and rapid reversibility of the action of the drug (i.e., the effect of the drug is not sustained after washout such that the normal function of the inhibited target kinase is recoverable). This double reversibility is fundamental for the dissection of heterosynaptic interactions in plasticity. These protocols also involve three independent stimulus pathways to a common pool of hippocampal neurons and exceptional control of temperature and other parameters of slice physiology to enable routine stable long-term recordings and their measurement in excess of $12 \mathrm{~h}$ (see Fig. 1).

The present study explores the potentially differential roles of several $\mathrm{Ca}^{2+} /$ calmodulin-dependent protein kinase (CaMK) 
family members in the duration of LTP. Although CaMKII regulates many neuronal functions (Erondu and Kennedy, 1985; Braun and Schulman, 1995; Yamauchi, 2005), various theoretical ideas and experimental lines of evidence suggest that its activation is necessary locally for the induction and expression of LTP at synapses of the CA3 to CA1 pathway of the hippocampus (Lisman and Goldring, 1988; Malenka et al., 1989; Malinow et al., 1989). A natural question is whether CaMKII may also possess a specific role in tag setting.

In contrast, in the cell soma, calcium entry triggers activation of CaMK kinase (CaMKK) activity that stimulates CaMKIV, a nuclear kinase capable of phosphorylating $\mathrm{Ca}^{2+} / \mathrm{cAMP}$-response element binding protein (CREB) that initiates the transcription of plasticity-related genes (Bito et al., 1996; Ho et al., 2000; Kang et al., 2001). Might the pathway involving CaMKK and CaMKIV be essential for the synthesis of PRPs? To test these ideas, we examined whether reversible inhibition of different CaM kinases can specifically interfere with either the setting of tags or the synthesis of PRPs (supplemental Fig. S1, available at www.jneurosci.org as supplemental material).

\section{Materials and Methods}

The animals' care and maintenance and all experimental procedures were performed in accordance with United Kingdom Home Office regulations. Male Wistar rats $(n=110)$ aged $7-8$ weeks were anesthetized with isoflurane and decapitated, and the brain was removed rapidly. Brain slices $(400 \mu \mathrm{m})$ of the dorsal hippocampus were sectioned as described previously (Leutgeb et al., 2003). The hippocampal slices rested on top of eight layers of lens tissue paper (Whatman 105) placed on top of the hard surface of the slice chamber (BSC2 Scientific Systems Design; Digitimer). This ensured that the slice tissue was well soaked in the lens tissue paper while its surface remained exposed to air. All the artificial CSF (aCSF) volume held in the chamber was contained in the $2 \mathrm{~cm}$ wide $\times 2.5 \mathrm{~cm}$ long layers of lens tissue paper and amounted to $0.475 \mathrm{ml}$ (i.e., dead volume in the chamber). All the experiments were run with the same low flow rate $(0.4 \mathrm{ml} / \mathrm{min})$ to minimize mechanical disruption of the recordings. Under these conditions, we calculate a full recycling of the aCSF every $71 \mathrm{~s}$ (i.e., 17 washes in $20 \mathrm{~min}$ ). Although every drug has its own characteristics (i.e., site of action, affinity), we judged that $20 \mathrm{~min}$ would be enough to wash out the drugs used in these experiments. When drug washouts could be critical, alternative experiments were used to corroborate the interpretation of the result. The aCSF was prepared with the following concentrations (in $\mathrm{mM}$ ): $124 \mathrm{NaCl}, 3.7 \mathrm{KCl}, 1.2 \mathrm{KH}_{2} \mathrm{PO}_{4}$, $1.0 \mathrm{MgSO}_{4}\left(7 \mathrm{H}_{2} 0\right), 2.5 \mathrm{CaCl}_{2}, 24.6 \mathrm{NaHCO}_{3}$, and $10 \mathrm{D}$-glucose, final $\mathrm{pH}$ $7.4 \pm 0.05$. The full rig, including all the electrode holders, was heated to $32^{\circ} \mathrm{C}$ via the ETC system (University of Edinburgh), a procedure that prevents condensation droplets falling onto the interface chamber slices and so affecting neuronal stability.

One recording electrode, made from stainless steel rods (712700 A-M Systems), and three monopolar stainless steel electrodes (571000 A-M Systems) stimulating electrodes were positioned in CA1 (Fig. 1A). The slices rested for at least $2 \mathrm{~h}$ before preliminary recordings were taken, and no high-frequency stimulation was delivered until $5 \mathrm{~h}$ after dissection. The importance of this resting period for the stabilization of kinase phosphorylation levels has been emphasized previously (Ho et al., 2004; Sajikumar et al., 2005). Field EPSPs (fEPSPs) were recorded at $40-50 \%$ of the maximum amplitude obtained in an input-output curve. Test stimulation was $0.0067 \mathrm{~Hz}, 1$ pulse per $150 \mathrm{~s}$, resulting in one of the three channels being stimulated every $50 \mathrm{~s}(0.02 \mathrm{~Hz})$. This low rate was chosen to allow the activity rates of kinases and other molecules to drop to a resting state between stimulation (Sajikumar et al., 2005). At this low rate of stimulation, hippocampal slices react more slowly to drugs such as anisomycin than at faster rates (Fonseca et al., 2006; Sajikumar et al., 2008). Also importantly, test stimulation activates NMDA receptors and the molecular cascades linked to it (Navakkode et al., 2007). Minimizing the impact of test stimulation is in the interest of recording stability (Schurr et al., 1986) as well as reproducibility of the data from experiment to experiment.

Data (fEPSP slope) was normalized to a baseline calculated over 60 min before the first tetanization. The "strong" tetanization protocol consisted of three trains of 100 pulses at $100 \mathrm{~Hz}$ delivered at 10 min intervals (300 pulses in total). The "weak" tetanization protocol was a theta burst consisting of four trains, at a $200 \mathrm{~ms}$ interval, consisting of five pulses at $100 \mathrm{~Hz}$ (20 pulses in total). Pathway independence was tested by using standard pair-pulse stimulation protocols that alternated between the two stimulating pathways placed in the stratum radiatum of CA1 $(50 \mathrm{~ms}$ interpulse interval). The experiment proceeded provided this procedure failed to show paired-pulse facilitation (PPF). Normal PPF was detected when PPS was delivered to either of the two pathways as would be expected when the same presynaptic fibers are recruited by both pulses (data not shown). In addition to this control for pathway independence, the induction of LTP in one pathway fail to induce any LTP on the other two pathways in all our experiments. Pathway convergence is assumed from the limited area of sensitivity of the extracellular recording electrode, from the layered anatomy of the hippocampus, and from the observation of heterosynaptic plasticity dependent on intracellular processes (Schwartzkroin and Wester, 1975; Andersen et al., 1977; Frey and Morris, 1997). 


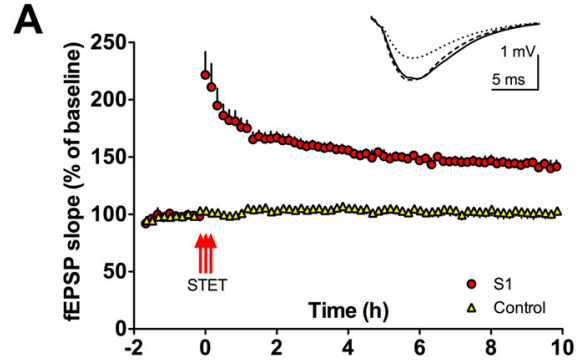

D

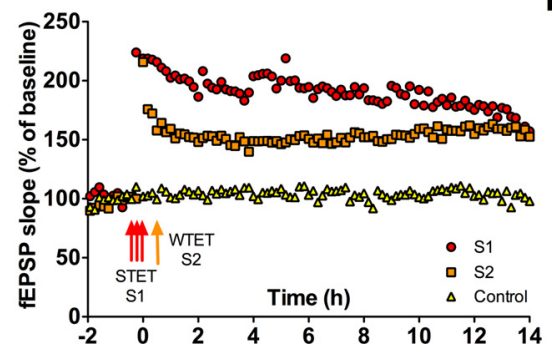

B

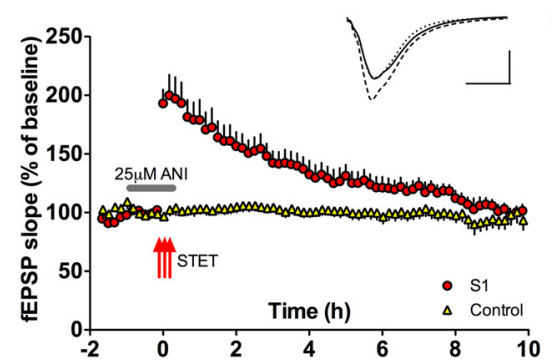

E

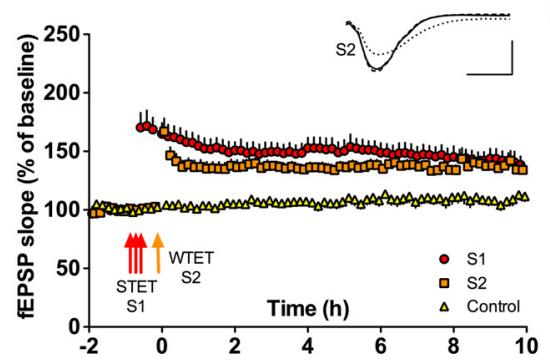

C

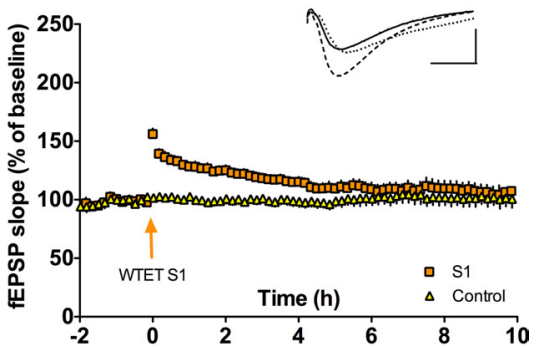

$\mathbf{F}$

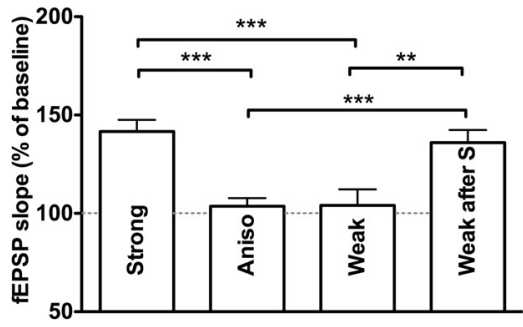

Figure 2. Synaptic tagging and capture in the stratum radiatum of CA1 pyramidal cells. $A$, Strong tetanization (STET) ( 3 trains of 100 pulses at $100 \mathrm{~Hz}$ given 10 min apart) produces an increase in the synaptic response that lasts $10 \mathrm{~h}$ (late-LTP) relative to both the pretetanization baseline $(t=8.0, p<0.01$; red symbols) and a nontetanized control pathway $(t=4.9, p<0.01$; yellow symbols). This strongly induced LTP was stable over time (comparison of 2 and $10 \mathrm{~h}$ time points; $t=2.0, p \geq 0.05$ ). The insets in each graph represent typical EPSP traces per stimulated input 30 min before (dotted line), $30 \mathrm{~min}$ after (broken line), and $10 \mathrm{~h}$ after (full line). Calibration: $1 \mathrm{mV}, 5 \mathrm{~ms}(n=7)$. $\boldsymbol{B}$, Late-LTP is not maintained when a strong tetanus is given in the presence of the protein synthesis blocker anisomycin. The potentiation declined to baseline after $10 \mathrm{~h}$ ( $\mathrm{S} 1 \mathrm{vs}$ baseline; $t=0.15, p \geq 0.05 ; n=9)$ ). C, A weak tetanus (WTET) ( 4 trains of 5 pulses at $100 \mathrm{~Hz}$ given 200 ms apart) elicits LTP present at $2 \mathrm{~h}$ ( $S 1$ vs control pathway; $t=4.3, p<0.01$ ) that returns to baseline strength after $3 \mathrm{~h}$ ( $S 1$ vs control pathway; $t=1, p \geq 0.05 ; n=6$ ). D,E, Early-LTP (orange symbols) is rescued into late-LTP ( 22 vs control pathway at $10 \mathrm{~h} ; t=3.4, p \leq 0.01$ ) when one set of synapses receives the weak tetanus 20 min after another set of synapses onto the same population of pyramidal cells has received a strong tetanus ( $\boldsymbol{D}$, single experiment; $\boldsymbol{E}$, pooled data; $n=8) . \boldsymbol{F}$, Bar graph showing differences in the level of potentiation $10 \mathrm{~h}$ after stimulation between the four different conditions presented in $\boldsymbol{A}-\boldsymbol{E}$ (one-way ANOVA, $F=15.13, p<0.001$ ). The change in synaptic efficacy remaining $10 \mathrm{~h}$ after the strong tetanization of a set of synapses is significantly higher than when the tetanization takes place under the presence of $25 \mu \mathrm{m}$ anisomycin $(t=5.45, p<0.001)$ and higher than the potentiation remaining after weak tetanization $(t=4.53, p<0.001)$. In a similar way, the potentiation left after $10 \mathrm{~h}$ in a pathway weakly stimulated before strong tetanization was given to an independent but convergent set of synapses is greater than if the weak tetanization is given alone $(t=3.92, p<0.01)$ or if a strong tetanus is given together with anisomycin $(t=4.81, p<0.001)$. Error bars indicate SEM. ${ }^{* *} p<0.01$; ${ }^{* * *} p<0.001$. Multiple $t$ tests were comparisons done with Bonferroni's correction.

KN-93 (2-[N-(2-hydroxyethyl)]- $N$-(4-methoxybenzenesulfonyl)amino$N$-(4-chlorocinnamyl)- $N$-methylbenzylamine), Myr-AIP (N-Myr-Lys-LysAla-Leu-Arg-Arg-Gln-Glu-Ala-Val-Asp-Ala-Leu-OH), and KN-92 (2-[N-(4'-methoxybenzenesulfonyl)] amino- $N$-(4'-chlorophenyl)-2-propenyl- $N$-methylbenzylamine phosphate) were obtained from Calbiochem, anisomycin and D-AP-5 were from Sigma, and STO-609 [7H-benzimidazo(2,1-a)benz(de)isoquinoline-7-one-3-carboxylic acid] was from Tocris Bioscience.

The average values of the slope function of the fEPSP (millivolts per milliseconds) for each time point were analyzed using paired (withingroup) and unpaired (between-group) $t$ tests; $p<0.05$ was considered as statistically significant, but we show more exacting levels of significance in many cases. Parametric tests were used because the data conformed to a Gaussian distribution, but analysis using nonparametric tests (MannWhitney and Wilcoxon's tests) supported the same conclusions. To measure the stability of late-LTP, we compared the absolute level of potentiation relative to the baseline $2 \mathrm{~h}$ after its induction with the level remaining after $10 \mathrm{~h}$. This measure distinguishes stability and amplitude in a manner that is not usually considered. Specifically, it distinguishes between "stable late-LTP" and "decaying LTP that still shows potentiation at the end of the experiment" because the latter may arise if there is strong initial expression of LTP. We hope that this measure clarifies the difference between strength and persistence of LTP.

Hippocampal dissociated cultures were prepared from neonatal Wistar rats and cultured on coverslips as described previously (Bito et al., 1996; Kawashima et al., 2009). At 20-21 d in vitro, at which synaptic network in the culture was well developed, neurons were silenced with 2 $\mu \mathrm{M}$ TTX for $2 \mathrm{~h}$ and treated with various concentrations of kinase inhibitors (KN-93 or STO-609 in 0.1\% DMSO) for 30 min in Tyrode's solution (in mM: $124 \mathrm{NaCl}, 2.5 \mathrm{KCl}, 1.0 \mathrm{NaH}_{2} \mathrm{PO}_{4}, 2.0 \mathrm{CaCl}_{2}, 2.0 \mathrm{MgCl}_{2}, 24.6$ $\mathrm{NaHCO}_{3}, 30$ D-glucose, and 20 HEPES, pH 7.4). The cultures were then stimulated with $10 \mu \mathrm{M}$ glutamate/100 $\mu \mathrm{M}$ glycine/ $1 \mu \mathrm{M}$ TTX in $0 \mathrm{Mg}^{2+}$
Tyrode's solution for $3 \mathrm{~min}$ in the presence of the inhibitors. After stimulation, neurons were immediately fixed in chilled methanol for $5 \mathrm{~min}$, followed by ice-cold $4 \%$ paraformaldehyde/4\% sucrose/PBS for $5 \mathrm{~min}$.

Immunocytochemistry was performed essentially as described previously (Kawashima et al., 2009). Briefly, the fixed cells were washed, permeabilized, and incubated in a blocking solution (3\% BSA/0.3\% Triton X-100/PBS) with a phosphatase inhibitor cocktail (PhosSTOP; Roche). The cells were then reacted with primary antibodies in the blocking solution. The primary antibodies used were anti-phosphorylated-CREB (pCREB) [rabbit monoclonal antibody (mAb); Epitomics] and anti-MAP2 (mouse mAb; Sigma) or anti-pCaMKII (rabbit polyclonal antibody; Promega) and anti-CaMKII $\alpha$ (mouse mAb; Invitrogen). After the wash, the primary antibodies were labeled with anti-mouse AlexaFluor488conjugated and anti-rabbit AlexaFluor594-conjugated secondary antibodies. The cells were then washed, stained with $4^{\prime}, 6^{\prime}$-diamidino-2phenylindole (DAPI), and mounted on slides.

Fluorescence images were acquired using a confocal laser scanning microscopy or an EM-CCD camera mounted on an inverted microscope. To obtain two-dimensional images, a $z$-stack of multiple confocal section images were acquired using an LSM510 META confocal laser microscope (Carl Zeiss), and then the stack data were projected into single planes by a summation algorithm. The projected images were color coded and shown. To quantify fluorescent pixel intensities, single-plane images were directly acquired using an EM-CCD camera (Andor) mounted on an inverted microscope (IX81; Olympus), to take advantage of the higher sensitivity and dynamic range of the camera. For quantification, regions of interest (ROIs) were set on neuronal nuclei, which were defined by DAPI and MAP2 staining, for pCREB immunoreactivity $(n=89-154$ neurons per condition), and on dendritic spines defined based on total CaMKII $\alpha$ immunoreactivity for pCaMKII $(n=127-203$ spines from 9-12 neurons). Average intensity in each ROI was calculated using the 
MetaMorph software (Universal Imaging Corporation), and dose-dependent curves were drawn using the Prism software (GraphPad Software). The values under TTX condition and stimulation without drugs (DMSO only) were defined as basal $(0 \%)$ and maximum $(100 \%)$ activities, respectively. The image analyses were done in a blind manner.

\section{Results}

Synaptic tagging and capture

Conventional experiments examining late-LTP relative to a nontetanized baseline cannot, alone, isolate the putatively distinct roles played by different signaltransduction pathways in synaptic tagging, the availability of PRPs, or the putative capture process. Two-pathway experiments deploying what we shall call "weakbefore-strong" and "strong-before-weak" protocols, in which weak and strong tetanization are given sequentially to two independent pathways, are essential to examine the dissociations that are central to this study. Ideally, to perform long experiments in which two independent pathways are tetanized at some point, it is desirable to have a three-pathway configuration with a third nontetanized pathway to monitor the overall stability of the slice preparation (Fig. 1). We incorporated this to enable potentiated pathways to be compared with the control pathway at various time points rather than only make comparisons to pretetanus baselines. We began by establishing that our tetanization protocols are successful in producing late-LTP that is exceptionally stable over time.

LTP lasting $>10 \mathrm{~h}$ with an absolute level of $\sim 142 \%$ (at $10 \mathrm{~h}$ ) relative to both the pretetanization baseline $(p<0.01)$ and higher than a nontetanized control pathway ( $p<0.01$ ) was routinely obtained after strong tetanization (Fig. 2A). This persistent late-LTP was sensitive to the presence of the protein synthesis blocker anisomycin at induction; in addition to displaying fEPSP decay over time with anisomycin, there was no difference between fEPSP slope at $10 \mathrm{~h}$ after tetanus (S1) and the pretetanus baseline $(p>$ $0.05)$ nor between $S 1$ and the control pathway at $10 \mathrm{~h}$ after tetanus ( $p \geq 0.05$ ). Using our novel "stability" measure (described in Materials and Methods), the within-pathway decline in the level of potentiation in S1 from 2 to $10 \mathrm{~h}$ was significant $(p<0.01$ ) (Fig. $2 B$ ). The NMDA receptor antagonist D-AP-5 $(25 \mu \mathrm{M})$ blocked LTP induction (S1 relative to control; $p>0.05)$. After washout and $1 \mathrm{~h}$ after the previous tetanus, a second strong tetanization was delivered to confirm the reversibility of the NMDA receptor block, and, under these conditions, late-LTP could be induced ( $p<0.05$ ) (supplemental Fig. S2, available at www.jneurosci.org as supplemental material). Error bars indicate SEM.
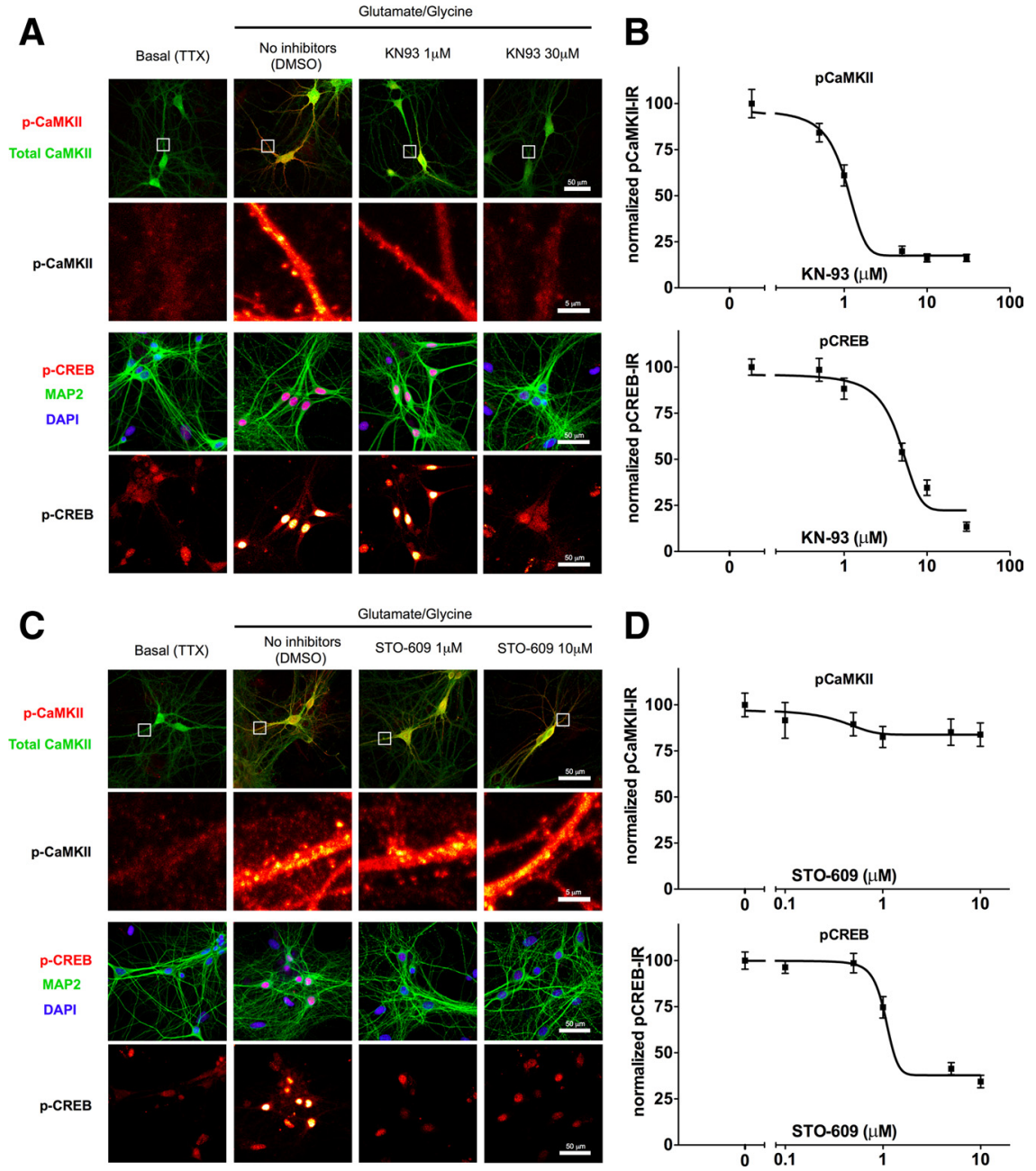

Figure 3. Dose-related effects of KN-93 and ST0-609 on phosphorylation of CaMKII and CREB. $\boldsymbol{A}$, Top, Effects of kinase inhibitors on pCaMKII (top rows) and on pCREB (bottom rows) in dissociated hippocampal culture. Neurons were stimulated with bath application of glutamate/glycine in the presence of KN-93. pCaMKII immunoreactivity in dendritic spines was measured for quantification. Framed areas in the top row were expanded and pCaMKII channel was shown at the bottom in a pseudocolor scale. pCREB immunoreactivity was quantified in neuronal nuclei that were identified with MAP2 and DAPI staining. The pCREB channel was separately shown in a pseudocolor scale below. $\boldsymbol{B}$, Differential dose responses of KN-93 on distinct CaMK pathways in culture neurons. Top, Effects of KN-93 on CaMKII autophosphorylation at Thr-286. Immunoreactivity (IR) for pCaMKII was quantified in dendritic spines and displayed as a function of KN-93 concentration. The ordinate represents basal (no stimulation, 0\%) to maximum (stimulated without inhibitors, 100\%) activities. Bottom, Effects of KN-93 on CREB phosphorylation at Ser-133. Suppression of pCREB immunoreactivity in the neuronal nuclei was displayed. Note greater sensitivity of KN-93 for CaMKII. C, Effects of ST0-609 on pCaMKII and on pCREB in dissociated hippocampal culture. Neurons were stimulated with bath application of glutamate/glycine in the presence of ST0-609. pCaMKII immunoreactivity in dendritic spines was measured for quantification. Framed areas in the top row were expanded and pCaMKII channel was shown at the bottom in a pseudocolor scale. pCREB immunoreactivity was quantified in neuronal nuclei that were identified with MAP2 and DAPI staining. The pCREB channel was separately shown in a pseudocolor scale below. D, Differential dose responses of ST0-609 on distinct CaMK pathways in culture neurons. Top, Effects of ST0-609 on CaMKII autophosphorylation at Thr-286. Bottom, Effects of STO-609 on CREB phosphorylation at Ser-133.

Thus, strong tetanization induced an NMDA receptordependent, anisomycin-sensitive late-LTP. Conversely, potentiation induced by weak tetanization was clearly present at $2 \mathrm{~h}$ ( $25 \%$ above baseline; $p<0.01$ ), but this early-LTP declined within $6 \mathrm{~h}$ and was absent after $10 \mathrm{~h}(p>0.05$, relative to control) (Fig. 2C). Using our measure of LTP stability over time, comparison of the 2 and $10 \mathrm{~h}$ time points showed a significant decline in the weakly tetanized synapses $(p<0.05)$.

To demonstrate STC, the strong tetanus protocol was then given to pathway S1, and, $20 \mathrm{~min}$ after the last $100 \mathrm{~Hz}$ train, a weak tetanus was applied to a second independent but conver- 
A

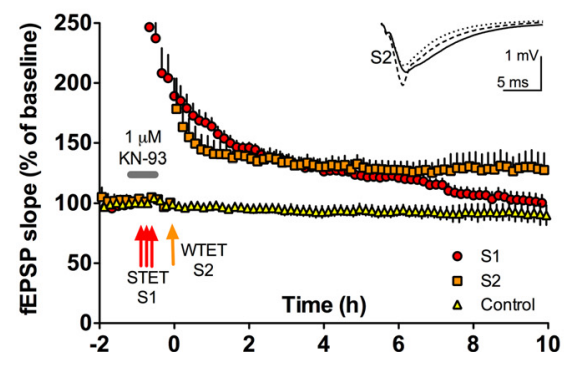

B

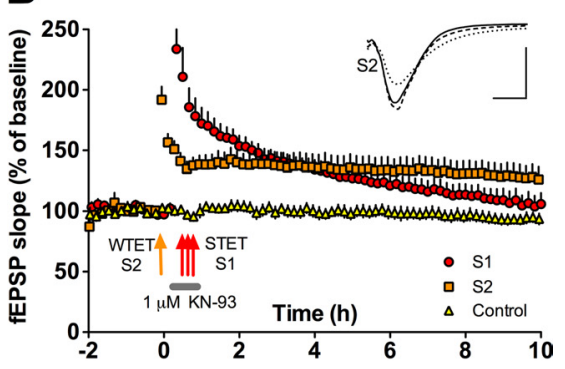

lies upstream of the CREB kinase CaMKIV but does not interfering with CaMKII phosphorylation (Fig. 3C,D).

KN-93 at $1 \mu \mathrm{M}$ blocks late-LTP but allows a separate and weakly tetanized pathway to display late-LTP

Using the critical strong-before-weak protocol, KN-93 $(1 \mu \mathrm{M})$ was applied during the strong tetanization of one pathway (S1, to block late-LTP) and washed out, and, 20 min later, weak tetanization was delivered to pathway S2. KN-93 completely blocked late-LTP on pathway S1 (compare pretetanus baseline at $10 \mathrm{~h}$ and control pathway in Fig. $4 A, p>0.05)$. In contrast, pathway S2 weakly stimulated 20 min after KN-93 washout maintained late-LTP for at least $10 \mathrm{~h}$ (Fig. $4 A, p<$ $0.05)$ and was stable from 2 to $10 \mathrm{~h}(p>$

gent pathway S2 (strong-before-weak protocol). In a single representative experiment, late-LTP was observed on both pathways lasting $>14 \mathrm{~h}$ (Fig. $2 D$ ). The group data of a series of experiments $(n=8)$ revealed that pathway S2 maintained its potentiated state for at least $10 \mathrm{~h}$. This was shown by comparing $\mathrm{S} 2$ with the third, control pathway ( $p \leq 0.01$ ) and by establishing stability over the $2-10 \mathrm{~h}$ posttetanization time period (S2 at 2 vs $10 \mathrm{~h}, p \geq 0.05$ ) (Fig. 2E). This stable late-LTP on S2 also differed from that seen on weakly tetanized pathways in slices that did not receive heterosynaptic strong stimulation (compare $\mathrm{S} 2$ in Fig. $2 E$ with $C$ at $10 \mathrm{~h}: t=3.4, p<0.05)$.

These results confirm previous findings (Frey and Morris, 1997, 1998a) and extend them through our use of three pathways and a monitoring time after LTP of $>8 \mathrm{~h}$, additional features essential for the later phases of experimentation.

\section{Differential sensitivity of phosphorylated CaMKII and CREB to varying concentrations of $\mathrm{KN}-93$ and STO-609}

The CaMK inhibitor KN-93 has a broad spectrum of specificity for inhibiting CaM kinases, but the dose-response profile differs between CaMK subtypes. To verify the sensitivity of both CaMKII-mediated and CaMKK/CaMKIV-mediated phosphorylation processes to $\mathrm{KN}-93$, cultured hippocampal neurons were exposed to robust glutamate-induced stimulation of phosphorylation, and we measured immunoreactivities for both pCaMKII (as a readout of CaMKII activity) and pCREB (as a functional readout of CaMKIV activation), in the presence of graded concentrations of KN-93 (Fig. 3A). Both pCaMKII and pCREB were blocked at a high concentration (e.g., $30 \mu \mathrm{M}$ ). The dose-response relation for inhibition of pCREB was rightward shifted relative to pCaMKII inhibition (Fig. 3B), consistent with the ideas that (1) CaMKII phosphorylation is more sensitive to $\mathrm{KN}-93$, and (2) a higher concentration of KN-93 should be necessary to fully block PRP synthesis and availability. It is important to note that a low (1 $\mu \mathrm{M})$ concentration of KN-93 has been shown previously to be sufficient to impair synaptic plasticity in brain slices (Hansel et al., 2006). CaMKII, with a $K_{\mathrm{i}}$ of $370 \mathrm{~nm}$ (Sumi et al., 1991), should be effectively blocked at this concentration, whereas the activity of other CaM kinases, such as CaMKIV, would be far less potently inhibited (Ishida et al., 1995).

In contrast, immunocytochemical assays using cultured neurons revealed that high concentrations of STO-609 $(>1 \mu \mathrm{M})$ are capable of blocking phosphorylation of CREB, because CaMKK
$0.05)$. The level of potentiation in the two pathways "crossed over." This is a critical observation, because it implies that PRPs must have been made available to synapses of the S2 pathway. Because S2 was weakly tetanized, the PRPs were likely upregulated by the strong tetanization of pathway S1. Given that pathway S1 decayed to baseline, it follows that $1 \mu \mathrm{M} \mathrm{KN}-93$ must have selectively blocked a process critical for late-LTP but distinct from PRP availability, namely tag setting.

We confirmed these findings using the alternative weakbefore-strong protocol. The weakly tetanized pathway (S2) showed sustained late-LTP over $10 \mathrm{~h}(p<0.05)$, despite the decay to baseline (and crossover) of the potentiation induced by the later strong tetanization in the presence of $\mathrm{KN}-93$ of the $\mathrm{S} 1$ pathway (compare baseline and control pathway in Fig. $4 B, p>$ 0.05). Together, these experiments suggest that CaMKII, being sensitive to lower concentrations of KN-93, might play a major role in tag setting, whereas its role in the regulation of PRP availability might be more limited.

This suggestion relies on the actions of a general CaMK inhibitor that was used at a low dose at which it preferentially inhibits CaMKII. To strengthen the view of a specific role for CaMKII in tag setting, we also tested the effect of a myristoylated, cellpermeant, form of a CaMKII-selective inhibitory peptide, AIP (Ishida et al., 1995). We first confirmed that Myr-AIP can strongly inhibit pCaMKII formation in cultured neurons in the absence of any effect on pCREB (supplemental Fig. S3 $A, B$, available at www.jneurosci.org as supplemental material). Next, using a standard strong-before-weak STC protocol, we tested whether the rescue of early-LTP into late-LTP (supplemental Fig. S3C, available at www.jneurosci.org as supplemental material) would be compromised by the presence of $5 \mu \mathrm{M}$ Myr-AIP during earlyLTP induction. Myr-AIP was bath applied during the weak tetanization of S2. In keeping with our previous results with a low concentration of KN-93, pathway S2 failed to show late-LTP [compare with baseline in supplemental Fig. S3D (available at www.jneurosci.org as supplemental material), $p>0.05$ ]. We also note that early-LTP is diminished in the presence of Myr-AIP, consistent with previous results (Sanhueza et al., 2007). This indicates that Myr-AIP blocked the ability of PRPs generated in pathway S1 to convert a subsequent early-LTP in S2 into lateLTP, i.e., it compromised tag setting. Together, these data 
indicate that CaMKII phosphorylation specifically mediates tag setting.

STO-609 blocks late-LTP but permits a pathway tetanized in its presence to display late-LTP provided an independent pathway is tetanized strongly

The CaMKK-CaMKIV pathway mediates CREB phosphorylation and neuronal activity gene transcription (Bito et al., 1996; Ho et al., 2000; Kang et al., 2001). However, it has not been formally addressed before whether a CaMKK-CaMKIV-pCREB pathway might contribute to the regulation of PRP availability. Three-pathway protocols were then used to examine the contribution to late-LTP the CaMKKCaMKIV-dependent pathway. We used the CaMKK inhibitor STO-609 (Tokumitsu et al., 2002). We first confirmed that STO$609(5 \mu \mathrm{M})$ blocks the late phase of LTP (S1 compared with baseline in Fig. $5 A, p>$ $0.05)$. Because STO-609 has little effect on CaMKII activity (Fig. 3C,D), our perspective is that STO-609 may block late-LTP through a selective effect on the synthesis or distribution of PRPs. To test this, we therefore made the drug present during the weak tetanization of pathway S2 and then washed it out before strong tetanization was applied to pathway S1. In this weak-beforestrong protocol, we observed a rescue of early-LTP into late-LTP on the weakly stimulated pathway S2 (compared with baseline in Fig. $5 B, p<0.05$ ). This was not attributable to some cryptic "potentiating" effect of STO-609, because giving this drug during weak tetanization of a single pathway was without effect on its decay to baseline (Fig. $5 C, p>0.05)$. Thus, STO-609 blocks lateLTP without effect on tag setting. The STC theory holds that it must be attributable to limiting the synthesis or availability of PRPs via inhibition of the CaMKK-dependent pathway. We tested this directly using a standard "strong-beforestrong" STC protocol in which STO-609 was present during the first strong tetanization but not during the second one. In support of our interpretation, pathway S1 showed late-LTP (compared with baseline in Fig. $5 D, p<0.01$ ). Supplemental experiments using a weakbefore-strong protocol with STO-609 present during strong tetanization revealed no late-LTP on the weakly stimulated pathway, also in keeping with this interpretation [supplemental Fig. S4 (available at www.jneurosci.org as supplemental material), $p>0.05$ ].

\section{KN-93 at $10 \mu \mathrm{M}$ has less selectivity and blocks both tag setting and PRPs}

We conducted three additional control studies. First, our cellculture data indicate that $\mathrm{KN}-93$ blocks the phosphorylation of CREB at high but not low concentrations (Fig. $3 A, B$ ). Accordingly, we can make the additional prediction that the relative selectivity of a $1 \mu \mathrm{M}$ concentration of KN-93 on tag setting would be lost if the concentration was increased to $10 \mu \mathrm{M}$. Specifically, a
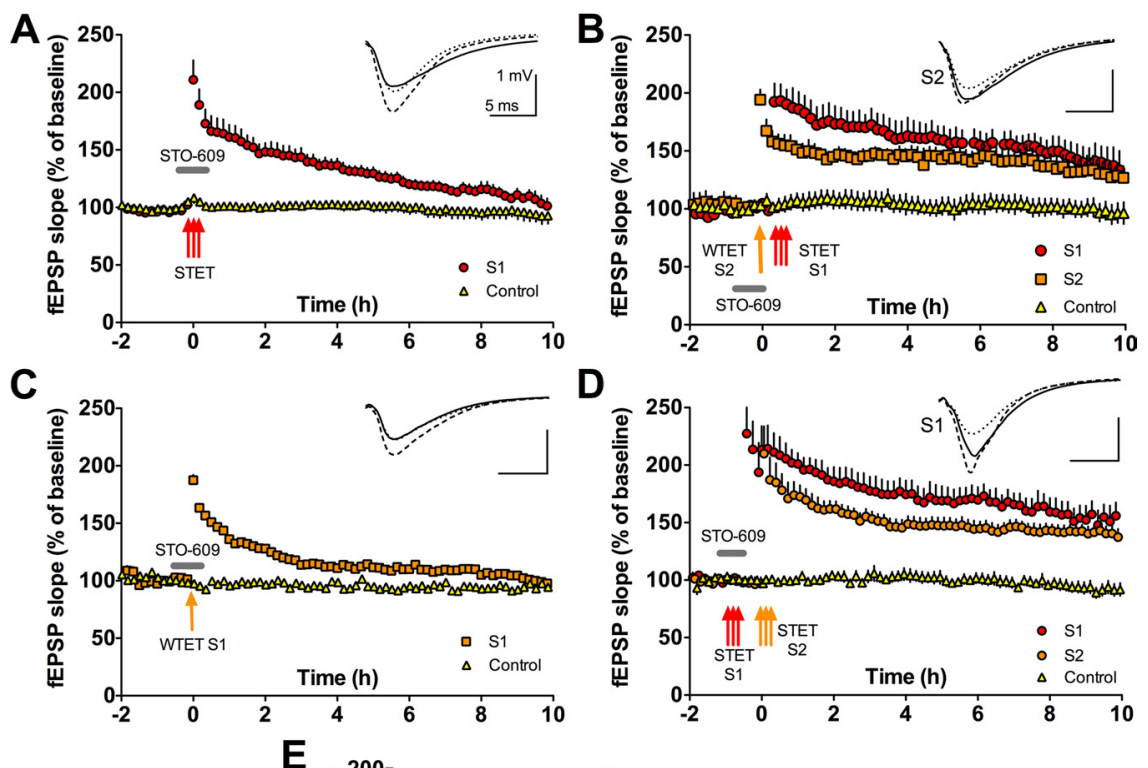

$E$

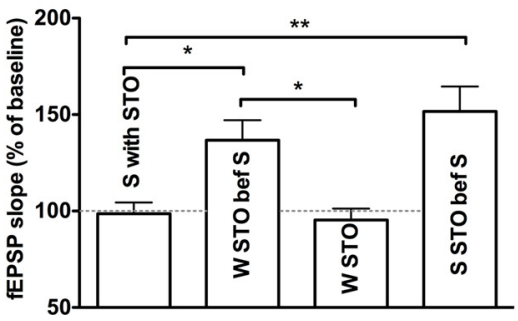

Figure 5. ST0-609 dissects a role for CaMKK in a cell-wide process necessary for late-LTP. $\boldsymbol{A}$, The CaMKK inhibitor ST0-609 (5 1 effect on a weakly tetanized pathway $S 1$ ( $S 1$ v baseline at $10 \mathrm{~h}, t=0.65, p>0.05 \cdot n=6$ ). $D$, In a strong-before-strong 作 level of potentiation $10 \mathrm{~h}$ after stimulation between the four different conditions presented in $\boldsymbol{A - D}$ (one-way ANOVA, $F=9.82$, 05) (1) tetanus with ST0-609 rescued by a strong tetanus, $t=4.17, p<0.01$ ). Error bars indicate SEM. ${ }^{*} p<0.05 ;{ }^{* *} p<0.01$. Symbols as in Figure 2. STET, Strong tetanization; WTET, weak tetanization.

higher concentration of KN-93 should block both tag setting and PRP synthesis and availability. Using the same strong-beforeweak protocol as the one described in Figure 4, $10 \mu \mathrm{M}$ KN-93 still blocked late-LTP of the strongly stimulated pathway S1 (compare baseline and control pathway in Fig. $6 A, p>0.05$ ), but pathway S2 that was weakly tetanized after KN-93 washout now also failed to maintain its potentiation (compare baseline and control pathway in Fig. $6 A, p>0.05$ ). The interpretation of this result relies on the complete washout of $\mathrm{KN}-93$ by the time the weak tetanus is delivered. As explained in Materials and Methods, our setup allows for $>15$ washes of the aCSF volume in the experimental chamber between the removal of the drug and the weak tetanization. So as not to rely only on the findings of Figure $4 C$, the same pattern of results was obtained using the weak-before-strong protocol, in which the weak tetanization is delivered before the application of KN-93 (10 $\mu \mathrm{M} \mathrm{KN-93} \mathrm{blocked} \mathrm{late-LTP} \mathrm{in} \mathrm{both} \mathrm{the}$ strong and weakly tetanized pathways) (Fig. $6 B, p>0.05$ ). A parsimonious explanation for this would be the contribution of 
A
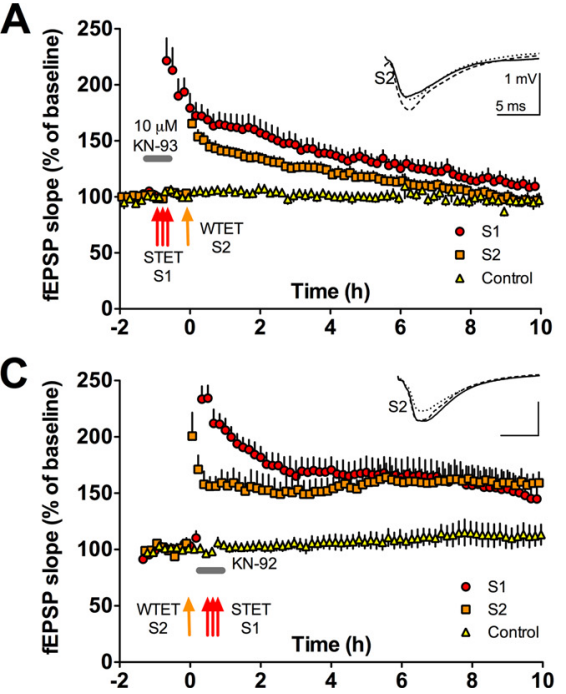

B

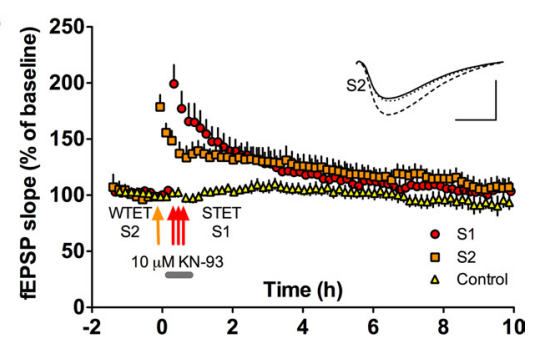

D

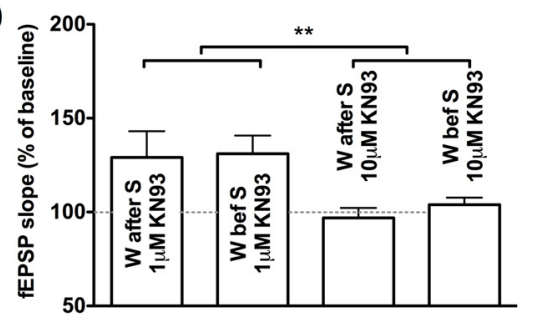

Figure 6. A higher concentration of KN-93 has less selective effects than a lower concentration. $A, B$, Higher concentrations of KN-93 fail to dissociate synapse-specific and cell-wide processes in late-LTP, in contrast to data in Figure 2 with $1 \mu \mathrm{M}$ KN-93. $A, A$ weakly stimulated pathway ( $S 2$ ) failed to show late-LTP( 2 vs control $10 \mathrm{~h}, t=0.1, p \geq 0.05$ ) when the stimulation was given 20 min after a strong tetanus to $\mathrm{S} 1$ if the higher concentration of $10 \mu \mathrm{M} \mathrm{KN}-93$ was present during LTP induction. The strongly stimulated pathway also fails to show late-LTP (S1 vs control $10 \mathrm{~h}, t=1.4, p \geq 0.05 ; n=9$ ). $\boldsymbol{B}$, The rescue of early-LTP into late-LTP is also not seen in $\mathrm{S} 2$ ( $\mathrm{S} 2 \mathrm{vs}$ control $10 \mathrm{~h}, t=1.7, p \geq 0.05$ ) if tetanization is given when $10 \mu \mathrm{m} \mathrm{KN}-93$ is present during S1. S1 also fails to maintain late-LTP (S1 vs control $10 \mathrm{~h}, t=0.9, p \geq 0.05 ; n=7$ ). C, The rescue of early-LTP into late-LTP in a weakly tetanized pathway (S2) is seen even if $\$ 1$ strong tetanization is delivered when KN-92 is present ( $S 2$ vs control $10 \mathrm{~h}$ after tetanus, $t=3.5, p<0.01 ; n=5$ ). $\boldsymbol{D}$, Bar graph showing differences in the level of potentiation $10 \mathrm{~h}$ after stimulation between the two concentrations of KN-93 used in Figures 4 and 6 (two-way ANOVA, $F=7.7, p<0.01$ ). The order of stimulation and drug application had no effect $(F=0.14, p>0.05)$. Error bars indicate SEM. ${ }^{* *} p<0.01$. Symbols as in Figure 2. STET, Strong tetanization; WTET, weak tetanization.

KN-93-sensitive CaM kinase(s) distinct from CaMKII, such as the CaMKK-CaMKIV pathway, with respect to PRP availability.

The second control study is for nonspecific effects of KN-93. We did this using the inactive analog KN-92 (10 $\mu \mathrm{M})$. Using a weak-before-strong protocol, KN-92 did not prevent the rescue of early-LTP on a weakly tetanized pathway S2 when given in the presence of strong tetanization on S1 (Fig. $6 C, p<0.01$ ).

Third, we checked that KN-93 would still block tag setting at a high concentration. In previous work (Sajikumar and Frey, 2007), a strong-before-strong protocol was used to examine the effect of KN-62 (1-[N,O-bis(5-isoquinolinesulphonyl)- $N$ methyl-L-tyrosyl]-4-phenylpiperazine) (an alternative inhibitor of CaM kinases) on late-LTP, finding evidence for a role in tag setting. The strong-before-strong protocol is sufficient to identify a role in tag setting, although not as analytically powerful as the strong-before-weak protocols. Nonetheless, we observed that, if present at the time of induction, a $10 \mu \mathrm{M}$ concentration of KN-93 blocked late-LTP at $10 \mathrm{~h}$ [pathway S2 compared with baseline in supplemental Fig. S5A (available at www.jneurosci.org as supplemental material), $p>0.05$ ] but had no effect on the induction of LTP on a pathway tetanized 20 min earlier [pathway $S 1$ compared with baseline in Fig. S5A (available at www.jneurosci.org as supplemental material), $p<0.01$ ]. In separate experiments, the order was reversed and the same dose of $\mathrm{KN}-93$ was present during the first tetanization but was then washed out. Late-LTP on the pathway tetanized under $10 \mu \mathrm{M} \mathrm{KN}-93$ failed to show any potentiation $10 \mathrm{~h}$ after tetanus when compared with a control baseline [pathway S1 in supplemental Fig. S5B (available at www. jneurosci.org as supplemental material), $p>0.05$ ]. However, after $20 \mathrm{~min}$ of aCSF flow into the chamber that successfully washed out KN-93, tetanization of the second pathway induced robust late-LTP lasting $10 \mathrm{~h}$ [pathway S2 in supplemental Fig.

$\mathrm{S} 5 B$ (available at www.jneurosci.org as supplemental material), $p<0.01]$. Also, KN-92 did not impair late-LTP if present during strong tetanization [compare baseline and control pathway in supplemental Fig. S5C (available at www. jneurosci.org as supplemental material), $p<0.01]$.

\section{Discussion}

These experiments have identified dissociable roles specific for distinct CaM kinase pathways in the persistence of synaptic potentiation, and they provide new molecular insights on the requirement for synapse-specific and cell-wide mechanisms mediating late-LTP. Our findings emerge from our use of long time course three-pathway protocols (i.e., two pathways tetanized in weak-before-strong and strong-before-weak modes, with a third nontetanized control pathway), which were readily combined with fast wash-in/ washout pharmacological treatments. First, we confirm previous observations (Frey and Morris, 1997; Fonseca et al., 2004) that early-LTP induced at one set of synapses can be rescued into late-LTP if, within a short time window (Frey and Morris, 1998a), late-LTP is induced at another set of synapses in the same CA1 neuronal population (Fig. 2). Second, cell-biological data reveals differential concentrationdependent effects of KN-93 and STO-609 on two complementary requirements for the maintenance of LTP, with $1 \mu \mathrm{M} \mathrm{KN}-93$ having specific effects on phosphorylation of CAMKII, whereas higher concentrations also affected pCREB, with STO-609 having no affect on pCaMKII (Fig. 3). Third, the low concentration of KN-93 selectively interrupted a pathway-specific tagging process but spared the availability of PRPs, as analytically revealed by successful late-LTP after weak tetanization of a second independent pathway (Fig. 4). A separate CaMKII inhibitor, Myr-AIP, had similar actions (supplemental Fig. S3, available at www. jneurosci.org as supplemental material). Fourth, STO-609 was shown to block late-LTP but to spare the setting of synaptic tags (Fig. 5). Fifth, consistent with dose-response profile shown in the cell-culture immunocytochemistry, we observed that a higher concentration of KN-93 blocked late-LTP but did so by both blocking tagging and other cell-wide mechanisms (Fig. 6), a result that builds on previous work with KN-62 (Sajikumar et al., 2007).

The synaptic tagging and capture hypothesis provides the key concepts that are central to our analysis of the rescue of early-LTP into late-LTP (Frey and Morris, 1998b). Indeed, this hypothesis asserts and predicts two requirements for long-term changes in synaptic efficacy: (1) the local setting of tags at stimulated synapses, and (2) the cell-wide or dendritic-domain-wide availability of PRPs that can be captured by the tags and so enable mechanisms responsible for the stabilization of potentiation (Reymann and Frey, 2007). However, the signal transduction pathways mediating these two requirements have remained unclear. Amid the potentially numerous molecular players and complex interactions involved in these processes, some may be 
required for both tag setting and $\mathrm{PRP}$ availability [e.g., activation of NMDA receptors (O'Carroll and Morris, 2004)]. Other molecules (or molecular states, such as phosphorylation) may only be necessary for tag setting and yet others only for PRP synthesis and/or their availability (supplemental Fig. S1, available at www. jneurosci.org as supplemental material). Our experiments identify a dissociable molecular cascade within the CaM kinase pathways for both tag setting and PRP supply.

\section{Specific targeting of tag setting using a low concentration of KN-93 and of PRP synthesis using STO-609}

CaMKII is a broad-range kinase that regulates many neuronal functions (Erondu and Kennedy, 1985; Braun and Schulman, 1995; Yamauchi, 2005). In the CA3 to CA1 Schaffer-collateral pathway of the hippocampus, CaMKII activation by $\mathrm{Ca}^{2+} / \mathrm{CaM}$ is required at the time of LTP induction (Malenka et al., 1989) and, as shown recently, remains necessary during the maintenance of LTP (Sanhueza et al., 2007). Compelling evidence indicates that a dodecameric holoenzyme is activated by intersubunit phosphorylation in the presence of calcium/calmodulin (Miller and Kennedy, 1986; Hanson et al., 1994). According to this model, during a robust $\mathrm{Ca}^{2+}$ signal, the binding of calmodulin to each subunit unmasks the kinase domain by repelling the autoinhibitory domain of CaMKII but also exposing a threonine 286 site (Thr-286) in the adjacent subunit. The subsequent phosphorylation of Thr-286 then generates an autonomous kinase activity and also increases the affinity for calmodulin and creating a state in which calmodulin is transiently trapped by CaMKII even shortly after the end of a $\mathrm{Ca}^{2+}$ transient (Meyer et al., 1992). It has been proposed that this property may enable active CaMKII to translocate into the spine (Zhang et al., 2008) and for autophosphorylated CaMKII to act as a switch capable of maintaining changes in synapse efficacy (Lisman and Goldring, 1988; Lisman and Zhabotinsky, 2001; Miller et al., 2005). This switch could, in principle, also participate in tag setting and/or the local capture of available PRPs (Sajikumar et al., 2007).

To secure definitive evidence for these ideas, we developed a long time course three-pathway slice recording protocol and then conducted robust differential CaMK pharmacological experiments whose aim was to experimentally dissociate tag setting and PRP supply. Our key observations are that (1) a low dose of $\mathrm{KN}-93$, which interferes with the phosphorylation of CaMKII in cell culture but spares most CREB phosphorylation, has the effect of specifically inhibiting tag setting while allowing PRP availability heterosynaptically, whereas (2) STO-609 selectively affects CREB phosphorylation but not pCaMKII and blocks PRP availability without affecting tag setting.

\section{The value of $10 \mathrm{~h}$ experiments and fast-reversible pharmacological interventions}

The present findings underwrite the value of allowing newly prepared brain slices, subject to minimal test stimulation, to stabilize for at least $4 \mathrm{~h}$ before tetanization, as long recommended by the Frey group in Magdeburg, Germany. Biochemical data suggest that it can take hours before kinase activation levels stabilize after slice preparation (Ho et al., 2004) and that low frequencies of test stimulation cause minimal interference with PRP availability (Fonseca et al., 2006). We also recorded for much longer periods than is typical in many LTP experiments, making it desirable, if not essential, to have a third nontetanized control pathway. Our data provide additional indications that the dynamic interactions set in train by tetanization continue for several hours (e.g., in Fig. $4 B$, the crossover of the strong and weakly tetanized pathways takes place over a period from 2 to $6 \mathrm{~h}$ after tetanization, and the strongly tetanized pathway does not decay to baseline until 8-10 h have elapsed). These points may seem merely methodological but, in our view, have been critical to dissecting the differential role of distinct CaM kinases with respect to synaptic tagging and capture.

It is unfortunate that we could not use brain slices from mice subject to gene targeting of the calcium/calmodulin pathway (Giese et al., 1998). The reason is that the dissociation of tag setting and PRP synthesis requires the differential inhibition of different components of CaMK pathways to be rapidly reversible. A pharmacological approach was essential, although we anticipate that our approach could be complemented by observations on suitable genetic reporter mice in the future.

\section{Multiple dissociable CaMK pathways contribute to STC}

Despite a large body of work on activity-dependent transcription/translation during long-term plasticity, surprisingly little has been elucidated on their relationship to the concrete implementation of long-lasting synaptic modifications. The evidence presented here suggests that STC may operate at least in part via two related yet distinct limbs of CaMK signaling: tag setting by CaMKII and activity-dependent gene transcription regulation by a CaMKK-dependent pathway. This new model nicely accounts for the interactive yet orthogonal (synapse-specific vs cell-wide) nature of tag setting and PRP synthesis, which might be coregulated in parallel via distinct pools of $\mathrm{Ca}^{2+} / \mathrm{CaM}$. However, the molecular basis of the tag-PRP interactions remains unclear. Better understanding of CaMKII dynamics (kinase activity and kinase content) and molecular dissection of the CaMKIIcontaining protein complexes at activated synapses will clearly be important steps toward solving this issue (Lisman et al., 2002; Yamauchi, 2005).

Because our data here establish the necessity for CaMKII activation to initiate the tag-setting process, one attractive idea is to consider that perhaps autophosphorylation of CaMKII itself might constitute the "tag" or be an integral part of it. However, independent studies have also shown evidence suggesting that pCaMKII signal may sometimes spread beyond the area of the originally stimulated synapses (Ouyang et al., 1997; Rose et al., 2009). The mechanistic basis for such potential loss of input specificity at the pCaMKII level remains unsolved. Additional studies are needed to resolve this apparent contradiction and pin down the molecular identity of the tag set in motion by CaMKII activation.

The wider implications of this work for the neurobiology of memory are of considerable interest. It provides a candidate mechanism for how inconsequential events (mimicked by weak stimulation in the protocols used here) may be remembered for much longer when they occur against the background of other surprising or emotionally significant events (mimicked by strong stimulation here), as occurs in flashbulb memory situations. Definitive evidence will require demonstration of STC in vivo and the creation of behavioral paradigms to investigate the transformation of short-term into long-term memory in complementary situations (Moncada and Viola, 2007).

\section{References}

Andersen P, Sundberg SH, Sveen O, Wigström H (1977) Specific longlasting potentiation of synaptic transmission in hippocampal slices. Nature 266:736-737.

Bito H, Deisseroth K, Tsien RW (1996) CREB phosphorylation and dephosphorylation: $\mathrm{a} \mathrm{Ca}^{2+}$ - and stimulus duration-dependent switch for hippocampal gene expression. Cell 87:1203-1214. 
Bliss TV, Lomo T (1973) Long-lasting potentiation of synaptic transmission in the dentate area of the anaesthetized rabbit following stimulation of the perforant path. J Physiol 232:331-356.

Braun AP, Schulman H (1995) The multifunctional calcium/calmodulindependent protein kinase: from form to function. Annu Rev Physiol 57:417-445.

Erondu NE, Kennedy MB (1985) Regional distribution of type II Ca ${ }^{2+}$ / calmodulin-dependent protein kinase in rat brain. J Neurosci 5:32703277.

Fonseca R, Nägerl UV, Morris RG, Bonhoeffer T (2004) Competing for memory: hippocampal LTP under regimes of reduced protein synthesis. Neuron 44:1011-1020.

Fonseca R, Nägerl UV, Bonhoeffer T (2006) Neuronal activity determines the protein synthesis dependence of long-term potentiation. Nat Neurosci 9:478-480.

Frey U, Morris RGM (1997) Synaptic tagging and long-term potentiation. Nature 385:533-536.

Frey U, Morris RGM (1998a) Weak before strong: dissociating synaptic tagging and plasticity-factor accounts of late-LTP. Neuropharmacology 37:545-552.

Frey U, Morris RGM (1998b) Synaptic tagging: implications for late maintenance of hippocampal long-term potentiation. Trends Neurosci 21:181-188.

Frey U, Krug M, Reymann KG, Matthies H (1988) Anisomycin, an inhibitor of protein synthesis, blocks late phases of LTP phenomena in the hippocampal CA1 region in vitro. Brain Res 452:57-65.

Giese KP, Fedorov NB, Filipkowski RK, Silva AJ (1998) Autophosphorylation at Thr286 of the alpha calcium-calmodulin kinase II in LTP and learning. Science 279:870-873.

Hansel C, de Jeu M, Belmeguenai A, Houtman SH, Buitendijk GH, Andreev D, De Zeeuw CI, Elgersma Y (2006) alphaCaMKII Is essential for cerebellar LTD and motor learning. Neuron 51:835-843.

Hanson PI, Meyer T, Stryer L, Schulman H (1994) Dual role of calmodulin in autophosphorylation of multifunctional CaM kinase may underlie decoding of calcium signals. Neuron 12:943-956.

Ho N, Liauw JA, Blaeser F, Wei F, Hanissian S, Muglia LM, Wozniak DF, Nardi A, Arvin KL, Holtzman DM, Linden DJ, Zhuo M, Muglia LJ, Chatila TA (2000) Impaired synaptic plasticity and cAMP response element-binding protein activation in $\mathrm{Ca}^{2+} / \mathrm{calmodulin}^{2}$ dependent protein kinase type IV/Gr-deficient mice. J Neurosci 20:6459-6472.

Ho OH, Delgado JY, O’Dell TJ (2004) Phosphorylation of proteins involved in activity-dependent forms of synaptic plasticity is altered in hippocampal slices maintained in vitro. J Neurochem 91:1344-1357.

Ishida A, Kameshita I, Okuno S, Kitani T, Fujisawa H (1995) A novel highly specific and potent inhibitor of calmodulin-dependent protein kinase II. Biochem Biophys Res Commun 212:806-812.

Kang H, Sun LD, Atkins CM, Soderling TR, Wilson MA, Tonegawa S (2001) An important role of neural activity-dependent CaMKIV signaling in the consolidation of long-term memory. Cell 106:771-783.

Kawashima T, Okuno H, Nonaka M, Adachi-Morishima A, Kyo N, Okamura M, Takemoto-Kimura S, Worley PF, Bito H (2009) Synaptic activityresponsive element in the Arc/Arg3.1 promoter essential for synapse-tonucleus signaling in activated neurons. Proc Natl Acad Sci U S A 106:316-321.

Krug M, Lössner B, Ott T (1984) Anisomycin blocks the late phase of longterm potentiation in the dentate gyrus of freely moving rats. Brain Res Bull 13:39-42.

Leutgeb JK, Frey JU, Behnisch T (2003) LTP in cultured hippocampalentorhinal cortex slices from young adult (P25-30) rats. J Neurosci Methods 130:19-32.

Lisman J, Schulman H, Cline H (2002) The molecular basis of CaMKII function in synaptic and behavioural memory. Nat Rev Neurosci 3:175-190.

Lisman JE, Goldring MA (1988) Feasibility of long-term storage of graded information by the $\mathrm{Ca}^{2+} /$ calmodulin-dependent protein kinase molecules of the postsynaptic density. Proc Natl Acad Sci U S A 85:5320-5324.

Lisman JE, Zhabotinsky AM (2001) A model of synaptic memory: a CaMKII/PP1 switch that potentiates transmission by organizing an AMPA receptor anchoring assembly. Neuron 31:191-201.
Malenka RC, Kauer JA, Perkel DJ, Mauk MD, Kelly PT, Nicoll RA, Waxham MN (1989) An essential role for postsynaptic calmodulin and protein kinase activity in long-term potentiation. Nature 340:554-557.

Malinow R, Schulman H, Tsien RW (1989) Inhibition of postsynaptic PKC or CaMKII blocks induction but not expression of LTP. Science 245:862-866.

Martin KC, Casadio A, Zhu H, Yaping E, Rose JC, Chen M, Bailey CH, Kandel ER (1997) Synapse-specific, long-term facilitation of aplysia sensory to motor synapses: a function for local protein synthesis in memory storage. Cell 91:927-938.

Meyer T, Hanson PI, Stryer L, Schulman H (1992) Calmodulin trapping by calcium-calmodulin-dependent protein kinase. Science 256:1199-1202.

Miller P, Zhabotinsky AM, Lisman JE, Wang XJ (2005) The stability of a stochastic CaMKII switch: dependence on the number of enzyme molecules and protein turnover. PLoS Biol 3:e107.

Miller SG, Kennedy MB (1986) Regulation of brain type II $\mathrm{Ca}^{2+}$ / calmodulin-dependent protein kinase by autophosphorylation: a $\mathrm{Ca}^{2+}$ triggered molecular switch. Cell 44:861-870.

Moncada D, Viola H (2007) Induction of long-term memory by exposure to novelty requires protein synthesis: evidence for a behavioral tagging. J Neurosci 27:7476-7481.

Navakkode S, Sajikumar S, Frey JU (2007) Synergistic requirements for the induction of dopaminergic D1/D5-receptor-mediated LTP in hippocampal slices of rat CA1 in vitro. Neuropharmacology 52:1547-1554.

O'Carroll CM, Morris RGM (2004) Heterosynaptic co-activation of glutamatergic and dopaminergic afferents is required to induce persistent long-term potentiation. Neuropharmacology 47:324-332.

Ouyang Y, Kantor D, Harris KM, Schuman EM, Kennedy MB (1997) Visualization of the distribution of autophosphorylated calcium/calmodulindependent protein kinase II after tetanic stimulation in the CA1 area of the hippocampus. J Neurosci 17:5416-5427.

Reymann KG, Frey JU (2007) The late maintenance of hippocampal LTP: requirements, phases, "synaptic tagging", "late-associativity" and implications. Neuropharmacology 52:24-40.

Rose J, Jin SX, Craig AM (2009) Heterosynaptic molecular dynamics: locally induced propagating synaptic accumulation of CaM kinase II. Neuron 61:351-358.

Sajikumar S, Navakkode S, Frey JU (2005) Protein synthesis-dependent long-term functional plasticity: methods and techniques. Curr Opin Neurobiol 15:607-613.

Sajikumar S, Navakkode S, Frey JU (2007) Identification of compartmentand process-specific molecules required for "synaptic tagging" during long-term potentiation and long-term depression in hippocampal CA1. J Neurosci 27:5068-5080.

Sajikumar S, Navakkode S, Frey JU (2008) Distinct single but not necessarily repeated tetanization is required to induce hippocampal late-LTP in the rat CA1. Learn Mem 15:46-49.

Sanhueza M, McIntyre CC, Lisman JE (2007) Reversal of synaptic memory by $\mathrm{Ca}^{2+} /$ calmodulin-dependent protein kinase II inhibitor. J Neurosci 27:5190-5199.

Schurr A, Reid KH, Tseng MT, Edmonds HL Jr, West CA, Rigor BM (1986) Effect of electrical stimulation on the viability of the hippocampal slice preparation. Brain Res Bull 16:299-301.

Schwartzkroin PA, Wester K (1975) Long-lasting facilitation of a synaptic potential following tetanization in the in vitro hippocampal slice. Brain Res 89:107-119.

Sumi M, Kiuchi K, Ishikawa T, Ishii A, Hagiwara M, Nagatsu T, Hidaka H (1991) The newly synthesized selective $\mathrm{Ca}^{2+} /$ calmodulin dependent protein kinase II inhibitor KN-93 reduces dopamine contents in PC12h cells. Biochem Biophys Res Commun 181:968-975.

Tokumitsu H, Inuzuka H, Ishikawa Y, Ikeda M, Saji I, Kobayashi R (2002) STO-609, a specific inhibitor of the $\mathrm{Ca}^{2+} /$ calmodulin-dependent protein kinase kinase. J Biol Chem 277:15813-15818.

Yamauchi T (2005) Neuronal $\mathrm{Ca}^{2+} /$ calmodulin-dependent protein kinase II- discovery, progress in a quarter of a century, and perspective: implication for learning and memory. Biol Pharm Bull 28:1342-1354.

Zhang YP, Holbro N, Oertner TG (2008) Optical induction of plasticity at single synapses reveals input-specific accumulation of $\alpha$ CaMKII. Proc Natl Acad Sci U S A 105:12039-12044. 